\section{Lactobacillus endocarditis with prosthetic material: a case report on non-surgical management with corresponding literature review}

\author{
Mena Botros, Deepa Mukundan \\ Department of Pediatrics, Division of \\ Infectious Diseases, University of Toledo \\ College of Medicine, Toledo, $\mathrm{OH}$, USA
}

\section{Abstract}

Lactobacilli are rod shaped gram positive bacteria that naturally colonize the human gastrointestinal and genitourinary tracts and occasionally cause disease in humans. Lactobacillus infections are found in patients who are immunocompromized or have severe comorbidities. We report Lactobacillus endocarditis in a 17-year-old adolescent girl with cardiac prosthetic material following surgical correction for complex cyanotic congenital heart disease. Accurate identification of the organism can be delayed. Despite in vivo susceptibility to vancomycin, our patient clinically failed vancomycin therapy but ultimately responded to a six-week course of penicillin, in addition to a 4-week course of clindamycin and gentamicin. She recovered without the need for surgical intervention and has been symptom free for one year. Upon review of the literature, we found that Lactobacillus endocarditis has not been reported in a pediatric patient with complex cyanotic congenital heart disease.

\section{Introduction}

Lactobacillus is a facultative gram-positive large rod that is a rare pathogen and is considered normal flora of the oral cavity, gastrointestinal tract, and female genitourinary tract. ${ }^{1,2}$ These bacteria are utilized in probiotic supplements to aid in the treatment of conditions such as antibiotic-associated diarrhea. ${ }^{3}$ Lactobacillus infections are almost always seen in patients who are immunocompromized and in those who have underlying structural abnormality of the heart with or without prosthetic material. Lactobacillus bacteremia, albeit rare, is more common than endocarditis. ${ }^{1-4} \mathrm{~A}$ history of dental manipulation can be elicited in $75 \%$ of patients with Lactobacillus endocarditis. ${ }^{5}$

Here we report a case of Lactobacillus endocarditis that was successfully treated without surgical intervention in a 17 year old adoles- cent girl with extensive cardiac prosthetic material following surgical correction of complex cyanotic congenital heart disease.

\section{Case Report}

Our patient was a 17-year-old adolescent girl with an extensive past medical history that included aortic atresia with a ventricular septal defect (VSD), atrial septal defect (ASD), an interrupted inferior vena cava with drainage to the left superior vena cava, heterotaxy with dextrocardia, ventricular ectopy, pulmonary hypertension and polysplenia. She had an equally extensive surgical history that included Norwood arch reconstruction, VSD closure and conduit revision, Rastelli repair of unroofed coronary sinus, pacemaker implantation for sick sinus syndrome and lastly a cardiac catheterization with a placement of a pulmonary artery stent. Her last cardiac procedure (placement of the pulmonary stent) was 20 months prior to this episode. Socially, she is an active well developed adolescent school girl, who has met all her developmental milestones at the appropriate times and is looking forward to graduating from high school to start college in a few months' time. She initially presented to her cardiologist with 2 weeks of intermittent fevers. The cardiologist confirmed that she was pale and had dusky nail beds. No new murmurs were heard and a transthoracic echocardiogram showed no vegetation. She later presented to her primary care physician for continuing fever and associated fatigue. Urine and blood cultures were negative for bacterial growth. Co-incidentally she had dysmenorrhea with menorrhagia for which her gynecologist started her on oral contraceptive therapy along with iron supplements for iron deficiency anemia that was confirmed by laboratory studies. Since she was still febrile and fatigued, now with a $4 \mathrm{~kg}$ weight loss in 4 weeks, she was hospitalized for further evaluation. She appeared pale, had night sweats, myalgia, and chest pain. Her last dental cleaning was 4 months prior, and was done with appropriate antibiotic prophylaxis. She did not have a history of excessive intake of dairy products, nor was she on any probiotics. Physical examination revealed a tired looking pale teenager who was hemodynamically stable without any respiratory problems. Laboratory studies showed a normal total white blood cell count with mild predominance of neutrophils without any immature forms. She was anemic, with iron deficiency and had normal electrolytes, renal function and hepatic function. A cardiac CT scan showed non-occlusive vegetation in the pulmonary artery (at the root of the conduit) and a pulmonary septic embolus in the left lower lobe. Subsequent transthoracic echocar-
Correspondence: Deepa Mukundan, Department of Pediatrics, Division of Infectious Diseases, University of Toledo College of Medicine, Suite 2300, 2222 Cherry Street, Toledo, OH 43608, USA. Tel.: +1.419.251.8039 - Fax: +1.419.251.7715

E-mail: Deepa.Mukundan@utoledo.edu

Key words: lactobacillus, endocarditis, prostheses.

Contributions: MB, reviewed chart, reviewed and analyzed literature, composed and edited the manuscript; DM, concept, revised the manuscript critically for important intellectual content.

Conflict of interests: the authors declare no potential conflict of interests.

Received for publication: 28 May 2014.

Revision received: 27 July 2014 .

Accepted for publication: 28 July 2014 .

This work is licensed under a Creative Commons Attribution NonCommercial 3.0 License (CC BYNC 3.0).

(C) Copyright M. Botros and D. Mukundan, 2014 Licensee PAGEPress, Italy

Infectious Disease Reports 2014; 6:5497

doi:10.4081/idr.2014.5497

diogram confirmed the vegetation in the pulmonary artery. Two peripheral blood cultures were drawn upon admission to the hospital, one of which grew gram-positive rod like bacteria after 76 hours (3.2 days). The blood culture on the second day since admission also grew gram-positive rod like bacteria after 107 hours (4.5 days). All subsequent blood cultures were reported as no growth. She was started on empiric antimicrobial therapy with ceftriaxone and vancomycin dosed appropriately for endocarditis; and gentamicin for synergy at $1 \mathrm{mg} / \mathrm{kg}$ every eight hours. She also received a 5 day course of azithromycin for positive Mycoplasma serology (IgG and IgM). On day 6 the microbiology laboratory reported a preliminary identification of the gram positive rod like bacterium as Arcanobacterium haemolyticum, and therefore gentamicin and vancomycin were discontinued, and she was continued on ceftriaxone.

However, she was re-hospitalized after 7 days, for persistent and now almost continuous low grade fever with occasional high grade spikes and associated night sweats. A transthoracic echocardiogram now showed the vegetation to be of the same size and the ESR and CRP had increased. At that time, which was about two weeks since antibiotics inception; the final identification of the gram positive rod like bacterium was reported to be Lactobacillus by Matrix-Assisted Laser Desorption and Ionization method. ${ }^{6}$ The organism could not be further speciated and the sus- 
ceptibility studies revealed the organism to be susceptible to ceftriaxone, clindamycin, daptomycin, erythromycin, gentamicin, linezolid, penicillin and vancomycin (Table 1). Due to high risk for morbidity and mortality with repeat cardiac surgery in our patient, aggressive antimicrobial therapy was pursued with full dose gentamicin $2.5 \mathrm{mg} / \mathrm{kg}$ every 8 hours, clindamycin $10 \mathrm{mg} / \mathrm{kg}$ every 6 hours and penicillin 24 million units (adult dose) as continuous infusion daily. Our patient improved clinically with her fever trending down. She was later discharged, and continued on the aforementioned regimen for 4 weeks. She became afebrile after week 4 of therapy, after which gentamicin and clindamycin were discontinued. Penicillin infusion was continued until the end of week 6 . The transthoracic echocardiogram 11 months after the onset of her illness shows the vegetation to be less than half its original size. One year later, our patient has remained asymptomatic, anemia has resolved, she has gained weight and is back to school.

\section{Discussion}

Infective endocarditis (IE) due to Lactobacillus is relatively uncommon, as compared to IE due to organisms such as HACEK (Haemophilus aphrophilus, Actinobacillus actinomycetemcomitans, Cardiobacterium hominis, Eikenella corrodens, and Kingella kingae). ${ }^{1,3,4,7}$ The most recent review of Lactobacillus infections was done by Cannon et al. in 2005. They report on 241 cases of Lactobacillus infections of which $54 \%$ were bacteremia and $30 \%$ were endocarditis. ${ }^{3}$ In their review, $63 \%$ of patients with Lactobacillus endocarditis have structural heart disease, such as history of valvular disease, prosthesis placement, $12 \%$ had history of IE, $47 \%$ had a dental source and $26 \%$ had systemic emboli. ${ }^{3}$ Our patient also had complex congenital heart disease with prosthetic material, which places her at high risk for
Lactobacillus endocarditis, and subsequent complications such as pulmonary septic emboli, as is consistent with the literature. In our review of the literature we found only a couple of pediatric patients with Lactobacillus endocarditis and none with similar complex congenital heart disease. ${ }^{2,3}$

Salvana et al., in their 2006 review of patients with endocarditis, report surgical intervention was needed as the definitive treatment in $34 \%$ of patients). ${ }^{2}$ Cannon et al. ${ }^{3}$ report that $20 \%$ of the patients with endocarditis received monotherapy with penicillin, another $20 \%$ received dual therapy with penicillin and an aminoglycoside, the median duration of therapy was six weeks and the mortality was $23 \% .^{3}$ They also found that $100 \%$ of the endocarditis isolates were susceptible to ciprofloxacin, $80 \%$ to clindamycin, $36 \%$ to gentamicin $27 \%$ to vancomycin and $65 \%$ to penicillin. ${ }^{3}$ Although vancomycin is a reasonable option for empiric therapy of suspected endocarditis, especially with documented gram-positive bacteremia, Lactobacillus is often documented to be resistant to such therapy. ${ }^{1,3,9,9}$ Our patient had a very susceptible strain in vitro, and clinically she responded well to a combination of penicillin, clindamycin and gentamicin. We chose to administer multiple antimicrobials in view of the high risk of mortality and morbidity related to surgical intervention in her. There are some more atypical reports of previously healthy patients with Lactobacillus endocarditis following ingestion of large amounts of dairy, ${ }^{3,10-12}$ probiotic supplements, ${ }^{12-15}$ and some following procedures such as colonoscopy and dilation and curettage..$^{16,17}$ These possibilities were excluded in our patient, as she did not have a history of heavy dairy consumption, probiotic supplementation, or any recent gastrointestinal or genitourinary procedures.

Mortality rates reported for Lactobacillus endocarditis include $23 \%$ and $27 \%{ }^{2,3}$ High mortality is attributed to the unique pathogenic potential of Lactobacilli. Lactobacilli have been found to aggregate platelets, bind both

Table 1. Lactobacillus susceptibility report.

\begin{tabular}{lcl}
\hline Antibiotic & MIC $(\mu \mathrm{g} / \mathrm{mL})$ & MIC interpretation \\
Ceftriaxone & 0.5 & Sensitive \\
Clindamycin & $\leq 0.03$ & Sensitive \\
\hline Daptomycin & 4 & Sensitive \\
Erythromycin & 0.5 & Sensitive \\
\hline Gentamicin & $\leq 0.12$ & Sensitive \\
Linezolid & 2 & Sensitive \\
\hline Penicillin & $\leq 0.03$ & Sensitive \\
Vancomycin & 1 & Sensitive
\end{tabular}

fibronectin and fibrinogen, and adhere to collagen types I and V, which compose the extracellular matrix of endothelial cells and have been shown to be present at sites of endothelial damage. ${ }^{18}$

\section{Conclusions}

In summary, we report a unique case of an 17-year-old adolescent girl with a significant past medical and surgical history of complex cyanotic congenital heart disease, who was cured with multiple antimicrobials that included penicillin infusion, gentamicin and clindamycin; and most importantly, without surgical intervention.

\section{References}

1. Brouqui P, Raoult D. Endocarditis due to rare and fastidious bacteria. Clin Microbiol Rev 2001;14:177-207.

2. Salvana EM, Frank M. Lactobacillus endocarditis: case report and review of cases reported since 1992. J Infect 2006;53:e510.

3. Cannon JP, Lee TA, Bolanos JT, Danziger LH. Pathogenic relevance of Lactobacillus: a retrospective review of over 200 cases. Eur J Clin Microbiol Infect Dis 2005;24:3140.

4. Husni RN, Gordon SM, Washington JA, Longworth DL. Lactobacillus bacteremia and endocarditis: review of 45 cases. Clin Infect Dis 1997;25:1048-55.

5. Sussman JI, Baron EJ, Goldberg SM, et al. Clinical manifestations and therapy of Lactobacillus endocarditis: report of a case and review of the literature. Rev Infect Dis 1986;8:771-6.

6. Farfour E, Leto J, Barritault M, et al. Evaluation of the andromas matrix-assisted laser desorption ionization-time of flight mass spectrometry system for identification of aerobically growing gram-positive bacilli. J Clin Microbiol 2012;50:27027.

7. Atkins MC, Nicolson L, Harrison GA, et al. Lactobacillus jensenii prosthetic valve endocarditis. J Infect 1990;21:322-4.

8. Antony SJ. Lactobacillemia: an emerging cause of infection in both the immunocompromised and the immunocompetent host. J Natl Med Assoc 2000;92:83-6.

9. Sloss JM, Cumberland NS. Deep seated infection due to Lactobacillus caseii. J R Army Med Corps 1993;139:25-6.

10. Ze-Ze L, Tenreiro R, Duarte A, et al. Case of aortic endocarditis caused by Lactobacillus casei. J Med Microbiol 
2004;53: 451-3.

11. Soleman N, Laferl H, Kneifel W, et al. How safe is safe? A case of Lactobacillus paracasei ssp. paracasei endocarditis and discussion of the safety of lactic acid bacteria. Scand J Infect Dis 2003;35:759-62.

12. Charteris WP, Kelly PM, Morelli L, Collins JK. Antibiotic susceptibility of potentially probiotic Lactobacillus species. J Food Prot 1998;61:1636-43.

13. Franko B, Vaillant M, Recule C, et al. Lactobacillus paracasei endocarditis in a consumer of probiotics. Med Mal Infect 2013;43:171-3.

14. Mackay AD, Taylor MB, Kibbler CC, Hamilton-Miller JM. Lactobacillus endocarditis caused by a probiotic organism. Clin Microbiol Infect 1999;5:290-2.

15. Presterl E, Kneifel W, Mayer HK, et al. Endocarditis by Lactobacillus rhamnosus due to yogurt ingestion? Scand J Infect Dis 2001;33:710-4.

16. Avlami A, Kordossis T, Vrizidis N, Sipsas NV. Lactobacillus rhamnosus endocarditis complicating colonoscopy. J Infect 2001;42: 283-5.

17. Suarez-Garcia I, Sanchez-Garcia A, Soler $\mathrm{L}$, et al. Lactobacillus jensenii bacteremia and endocarditis after dilatation and curettage: case report and literature review. Infection 2012;40:219-22.

18. Harty DW, Oakey HJ, Patrikakis M, et al. Pathogenic potential of lactobacilli. Int $\mathrm{J}$ Food Microbiol 1994;24:179-89. 